\title{
A direct tying loop of silk sutures on the electrode
}

\author{
Ji Hee Hong, and Sae Young Kim \\ Department of Anesthesia and Pain Medicine, Keimyung University School of Medicine, Daegu, Korea
}

Editor, we experienced a case of lead migration in spinal cord stimulation (SCS), which was successfully corrected and fixed by tying the loops of silk sutures directly around the lead.

A-42-year-old man visited our pain clinic with a complaint of burning pain, causalgia, and paresthesia in both legs. Despite continuous conservative therapy, severe pain persisted in the lower back, both buttocks, and knee and ankle areas; therefore, we decided to try spinal cord stimulation.

After confirmation of induced paresthesia at the painful site, the lead and extension cables were connected, and a cylindrically shaped hard plastic was used for anchoring. Suture tying on the middle part of the cylindrically shaped hard plastic was performed with black silk. On the second day during the lead trial, paresthesia of the left leg disappeared; however, paresthesia developed in the lower abdomen. Although we changed the programming, paresthesia never developed at the painful site again. We suspected migration of the lead and checked the position of the lead through a T-L spine X-ray. On the left side, migration of the lead occurred in the cephalad direction, and the lead was positioned at the middle of the T7 level. To correct the position of the lead, we pulled the lead to the middle of the ninth vertebral body. Anchoring was performed again, and suture tying on the middle part of the cylindrically shaped hard plastic was performed with black silk. In addition, we made direct tying loops of silk sutures around the lead at the proximal site of the anchoring to prevent lead migration in the longitudinal direction again (Fig. 1). After revision of the lead, the patient could feel the paresthesia again on the painful side and the lead's position was maintained without any migration.

Lead migration results in a loss of proper paresthesia coverage and a subsequent reduction in pain relief. Andersen [1], reporting on the use of SCS for angina, found that the most frequent complication requiring repeated surgery was lead migration (23\%). Introduction of multichannel leads has greatly reduced the need for repeated revision as the result of lead migration. North et al. [2] found that programmable multichannel systems have a significantly greater clinical reliability than single-channel systems. Our patient also used multipolar leads; however, despite repeated trials of reprogramming, we failed in re-generation of paresthesia. When we checked the position of the lead, the lead had moved almost two vertebral levels, which is too far for generation of paresthesia to the painful site.

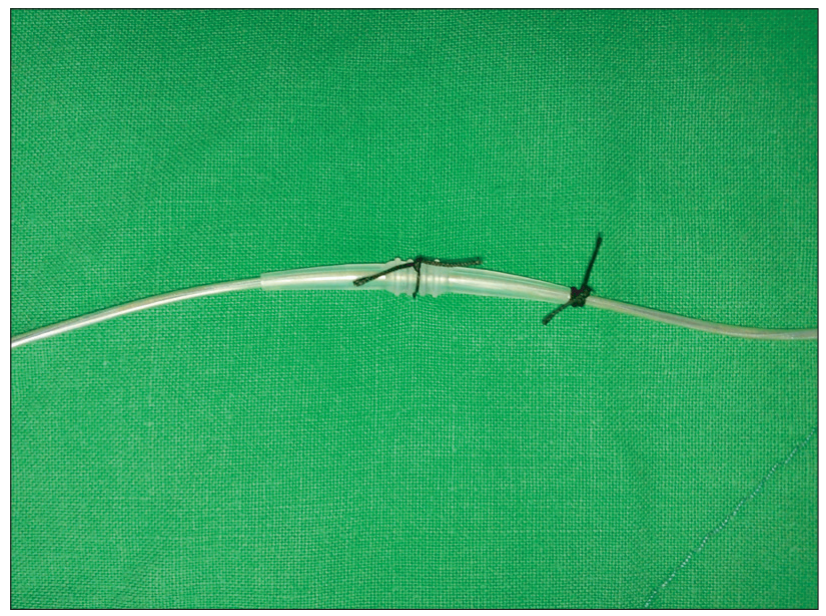

Fig. 1. This picture shows the tying loops of the silk suture directly around the lead.

Corresponding author: Ji Hee Hong, M.D., Ph.D., Department of Anesthesia and Pain Medicine, Keimyung University School of Medicine, 56, Dalseong-ro, Jung-gu, Daegu 700-712, Korea. Tel: 82-53-250-7288, Fax: 82-53-250-7240, E-mail: pain1004@dsmc.or.kr

(c) This is an open-access article distributed under the terms of the Creative Commons Attribution Non-Commercial License (http:// creativecommons.org/licenses/by-nc/3.0/), which permits unrestricted non-commercial use, distribution, and reproduction in any medium, provided the original work is properly cited. 
In this case, to prevent lead migration, we made direct tying loops of silk sutures around the lead at the proximal site of the anchoring, and the result was successful. Oh et al. [3] presented an alternate anchoring technique, which involves tying loops of silk sutures directly around the lead without the use of silicone or hard plastic anchors. In our case, we used the cylindrically shaped anchor; in addition, we tied loops of silk sutures directly around the lead.

Because the silk suture is tied directly on the lead, there is a possibility of lead damage. Kreis et al. [4] studied SCS lead integrity when direct silk sutures were made with supramaximal tension on the lead. They concluded that 28 SCS leads with 56 knot ties revealed no significant damage to polyurethane insulation by high power microscopy or change in conductor impedance. Because implanted silk sutures show a gradual loss of tensile strength over time, the risk of further wear and tear due to silk suture tension is minimal [4]. Our method has a limitation of being the routine for use in prevention of lead migration; however, it would be an alternative until a more successful and safe method can be developed.

\section{References}

1. Andersen C. Complications in spinal cord stimulation for treatment of angina pectoris. Differences in unipolar and multipolar percutaneous inserted electrodes. Acta Cardiol 1997; 52: 325-33.

2. North RB, Ewend MG, Lawton MT, Piantadosi S. Spinal cord stimulation for chronic, intractable pain: superiority of "multi-channel" devices. Pain 1991; 44: 119-30.

3. Oh MY, Ortega J, Bellote JB, Whiting DM, Alo K. Peripheral nerve stimulation for the treatment of occipital neuralgia and transformed migraine using a C1-2-3 subcutaneous paddle style electrode: a technical report. Neuromodulation 2004; 7: 103-12.

4. Kreis PG, Fishman SM, Chau K. Impact to spinal cord stimulator lead integrity with direct suture loop ties. Pain Med 2009; 10: 495500. 